PROCEEDINGS OF THE

AMERICAN MATHEMATICAL SOCIETY

Volume 132, Number 3, Pages 745-754

S 0002-9939(03)07103-X

Article electronically published on July 16, 2003

\title{
THE BACKWARD SHIFT ON THE SPACE OF CAUCHY TRANSFORMS
}

\author{
JOSEPH A. CIMA, ALEC MATHESON, AND WILLIAM T. ROSS
}

(Communicated by Joseph A. Ball)

\begin{abstract}
This note examines the subspaces of the space of Cauchy transforms of measures on the unit circle that are invariant under the backward shift operator $f \rightarrow z^{-1}(f-f(0))$. We examine this question when the space of Cauchy transforms is endowed with both the norm and weak* topologies.
\end{abstract}

\section{INTRODUCTION AND PRELIMINARIES}

In this note, we will examine the invariant subspaces of the backward shift operator

$$
(B f)(z)=\frac{f(z)-f(0)}{z}
$$

on the space of Cauchy transforms $\mathcal{K}$ consisting of analytic functions on the open unit disk $\mathbb{D}=\{z \in \mathbb{C}:|z|<1\}$ that take the form

$$
(K \mu)(z):=\int_{\mathbb{T}} \frac{d \mu(\zeta)}{1-\bar{\zeta} z} .
$$

Here $\mu \in M$, the space of finite Borel measures on the unit circle $\mathbb{T}=\{z \in \mathbb{C}$ : $|z|=1\}$.

By an "invariant subspace" of $\mathcal{K}$ we will mean a closed linear manifold $\mathcal{M} \subseteq \mathcal{K}$ for which $B \mathcal{M} \subseteq \mathcal{M}$. In using the word "closed", there are two topologies on $\mathcal{K}$ to consider here. The first is the norm topology. For $f \in \mathcal{K}$, let

$$
M_{f}:=\{\nu \in M: f=K \nu\}
$$

be the set of "representing measures" for $f$. Define the norm of an element $f \in \mathcal{K}$ by

$$
\|f\|:=\inf \left\{\|\nu\|: \nu \in M_{f}\right\},
$$

where $\|\nu\|$ denotes the total variation norm of the measure $\nu$. The notation $(\mathcal{K},\|\cdot\|)$ will denote the space $\mathcal{K}$ endowed with the above norm topology. It is well known that $(\mathcal{K},\|\cdot\|)$ is isometrically isomorphic to the quotient space $M / \overline{H_{0}^{1}}$ and is a non-separable Banach space. Here $H^{1}$ is the usual Hardy space of the disk [9] and $H_{0}^{1}$ are the functions in $H^{1}$ that vanish at the origin. $\overline{H_{0}^{1}}$ is regarded as a subspace of $M$ in the natural way as $\left\{\bar{f} d m: f \in H_{0}^{1}\right\}$ where $d m=|d \zeta| / 2 \pi$ is normalized Lebesgue measure on the circle. The second topology on $\mathcal{K}$ is the weak* topology

Received by the editors October 10, 2002 and, in revised form, October 21, 2002.

2000 Mathematics Subject Classification. Primary 46E15, 47A15; Secondary $47 \mathrm{~A} 16$.

Key words and phrases. Cauchy transforms, backward shift operator. 
that arises by identifying the dual space of the disk algebra $A$ (analytic functions on $\mathbb{D}$ that have continuous extensions to $\mathbb{D}^{-}$) with $\mathcal{K}$ via the pairing

$$
\langle f, K \mu\rangle=\int_{\mathbb{T}} \bar{f} d \mu, \quad f \in A, \mu \in M .
$$

By the F. and M. Riesz theorem [9] p. 41], if $\mu_{1}, \mu_{2} \in M_{K \mu}$, then $d \mu_{1}-d \mu_{2}=\bar{h} d m$, where $h \in H_{0}^{1}$. Thus the above pairing is independent of the representing measure $\mu$. We will use the notation $(\mathcal{K}, *)$ to denote the space $\mathcal{K}$ endowed with the weak ${ }^{*}$ topology. One can show that $(\mathcal{K}, *)$ is separable. Furthermore, every weak ${ }^{*}$ closed subspace of $\mathcal{K}$ is norm closed. See [4], 5], and [6] for a review of these basic facts about $\mathcal{K}$. In this paper, we examine the $B$-invariant subspaces of $(\mathcal{K}, *)$ and $(\mathcal{K},\|\cdot\|)$.

To put our results in perspective, we mention some known results about the $B$ invariant subspaces for other spaces of analytic functions. For example, by Beurling's theorem [9, p. 114], the $B$-invariant subspaces of the classical Hardy space $H^{2}$ all take the form $\left(\vartheta H^{2}\right)^{\perp}$, where $\vartheta$ is an inner function. Moreover [8] (see also [6]), $f$ belongs to $\left(\vartheta H^{2}\right)^{\perp}$ if and only if there is a function $G_{f} \in N^{+}\left(\mathbb{D}_{e}\right)$ that vanishes at infinity such that

$$
\lim _{r \rightarrow 1^{-}} \frac{f}{\vartheta}(r \zeta)=\lim _{r \rightarrow 1^{-}} G_{f}(\zeta / r)
$$

for $m$-almost every $\zeta \in \mathbb{T}$. Here $\mathbb{D}_{e}:=\widehat{\mathbb{C}} \backslash \mathbb{D}^{-}$and $G_{f} \in N^{+}\left(\mathbb{D}_{e}\right)$ means $G_{f}(1 / z) \in$ $N^{+}$(the Smirnov class of $\mathbb{D}\left[9\right.$, p. 25]). The function $G_{f}$ is called a "pseudocontinuation'] $[1]$ of $f$. If

$$
\sigma(\vartheta):=\left\{z \in \mathbb{D}^{-}: \varliminf_{\lambda \rightarrow z}|\vartheta(\lambda)|=0\right\},
$$

then, by basic properties of inner functions [11, pp. 68 and 69], $\vartheta$ has an analytic continuation to $\widehat{\mathbb{C}} \backslash \sigma(\vartheta)^{*}$, where $\sigma(\vartheta)^{*}:=\{z \in \widehat{\mathbb{C}}: 1 / \bar{z} \in \sigma(\vartheta)\}$. In fact, every $f \in\left(\vartheta H^{2}\right)^{\perp}$ has an analytic continuation to $\widehat{\mathbb{C}} \backslash \sigma(\vartheta)^{*}[8]$.

For the Bergman space $L_{a}^{2}$ (analytic functions $f$ on $\mathbb{D}$ such that $f \in L^{2}(d x d y)$ ) a theorem of Richter and Sundberg [14] says that every $B$-invariant subspace takes the form $\mathcal{M}_{g}:=\left\{f \in L_{a}^{2}: f \perp z^{n} g \forall n \in \mathbb{N} \cup\{0\}\right\}$ for some $g$ in the Dirichlet space (i.e., $g^{\prime} \in L_{a}^{2}$ ). Here we equate the dual of $L_{a}^{2}$ with the Dirichlet space via the "Cauchy" dual pairing

$$
\lim _{r \rightarrow 1^{-}} \int f(r \zeta) \overline{g(r \zeta)} d m(\zeta)
$$

Furthermore, (i) $g \mathcal{M}_{g} \subseteq H^{p}$ for all $0<p<1$, (ii) for every $f \in \mathcal{M}_{g}$, the meromorphic function $f / \vartheta_{g}$ (where $\vartheta_{g}$ is the inner factor of $g$ ) has a pseudocontinuation as in (1.2), (iii) every $f \in \mathcal{M}_{g}$ has an analytic continuation to $\widehat{\mathbb{C}} \backslash \sigma(g)^{*}$. Moreover [2], if $g$ is "sufficiently smooth", then $g \mathcal{M}_{g} \subseteq H^{1}$ and $f \in L_{a}^{2}$ belongs to $\mathcal{M}_{g}$ if and only if (a) $f g \in H^{1}$ and (b) $f / \vartheta_{g}$ has pseudocontinuation as in (1.2). For certain $L^{p}$ Bergman spaces, the function $g$ can always be chosen to be "sufficiently smooth"; so in this case we have a complete characterization of the $B$-invariant subspaces. Our purpose here is to get similar-looking results for the space $(\mathcal{K}, *)$ (which can be gleaned from results of Korenblum [13]) and to examine the more difficult problem of characterizing the $B$-invariant subspaces of $(\mathcal{K},\|\cdot\|)$.

\footnotetext{
${ }^{1}$ If $h$ is meromorphic on $\mathbb{D}$ and $H$ is meromorphic on $\mathbb{D}_{e}$ and the nontangential boundary values of $h$ and $H$ exist and are equal $m$-almost everywhere, then $h$ and $H$ are "pseudocontinuations" of each other. See [15] for more details.
} 


\section{The MAIN RESUlts}

For a $B$-invariant subspace $\mathcal{M}$ of $(\mathcal{K}, *)$ let

$$
\mathcal{M}_{\perp}=\{f \in A:\langle f, K \mu\rangle=0 \text { for all } K \mu \in \mathcal{M}\}
$$

be the pre-annihilator of $\mathcal{M} . \mathcal{M}_{\perp}$ is a norm closed subspace of the disk algebra $A$. A straightforward calculation shows that

$$
\langle f, K \mu\rangle=\int_{\mathbb{T}} \bar{f} d \mu=\lim _{r \rightarrow 1^{-}} \int_{\mathbb{T}} \overline{f(\zeta)}(K \mu)(r \zeta) d m(\zeta)=\lim _{r \rightarrow 1^{-}} \sum_{n=0}^{\infty} \overline{\widehat{f}(n)} \widehat{\mu}(n) r^{n}
$$

and $\langle f, B K \mu\rangle=\langle f, K(\bar{\zeta} d \mu)\rangle=\langle z f, K \mu\rangle$. Thus $z \mathcal{M}_{\perp} \subseteq \mathcal{M}_{\perp}$ since $B \mathcal{M} \subseteq \mathcal{M}$. Since $A$ is a Banach algebra and polynomials are dense in $A$ [11, p. 17], $\mathcal{M}_{\perp}$ is an ideal of $A$. A theorem of Rudin [16] (see also [11, p. 85]) says the following.

Theorem 2.2 (Rudin). Let I be a norm closed ideal of the disk algebra A. Then there is a closed set $E \subseteq \mathbb{T}$ of Lebesgue measure zero and an inner function $\vartheta$ with $\sigma(\vartheta) \cap \mathbb{T} \subseteq E$ such that

$$
I=I(\vartheta, E):=\left\{f \in A: f / \vartheta \in H^{\infty}, f \mid E=0\right\} .
$$

Furthermore, given a set $E \subseteq \mathbb{T}$ of Lebesgue measure zero and an inner $\vartheta$ with $\sigma(\vartheta) \cap \mathbb{T} \subseteq E$, there is an outer function $F \in A$ whose zero set is equal to $E$ and such that $g:=\vartheta F$ generates $I(\vartheta, E)$ in the sense that the smallest norm closed ideal of $A$ containing $g$ is equal to $I(\vartheta, E)$.

To describe $\mathcal{M}$, we need (via the Hahn-Banach theorem) to describe the set

$$
\left(\mathcal{M}_{\perp}\right)^{\perp}=I(\vartheta, E)^{\perp}:=\{f \in \mathcal{K}:\langle h, f\rangle=0 \text { for all } h \in I(\vartheta, E)\},
$$

or equivalently, the set $\left\{f \in \mathcal{K}:\left\langle z^{n} g, f\right\rangle=0 \forall n \in \mathbb{N} \cup\{0\}\right\}$. Korenblum [13] proved the following.

Theorem 2.3 (Korenblum). If $K \mu \perp I(\vartheta, E)$, then $K \mu$ has an analytic continuation to the set $\widehat{\mathbb{C}} \backslash\left(\sigma(\vartheta)^{*} \cup E\right)$.

In the process of proving our main theorem (Theorem 2.5), we will give an alternate proof of Korenblum's theorem. Any measure $\mu \in M$ can be decomposed uniquely as

$$
d \mu=\phi d m+d \mu_{s},
$$

where $\phi \in L^{1}(m)$ and $\mu_{s} \perp m$. Our main theorem describes $I(\vartheta, E)^{\perp}$.

Theorem 2.5. For $\mu \in M, K \mu \perp I(\vartheta, E)$ if and only if

(1) the support of $\mu_{s}$ is contained in $E$;

(2) $K \mu / \vartheta$ has an analytic continuation across $\mathbb{T} \backslash E$ to a function $F \in N^{+}\left(\mathbb{D}_{e}\right)$ with $F(\infty)=0$.

By the F. and M. Riesz theorem, every measure $\nu \in M_{f}(f \in \mathcal{K})$ has the same singular part. Thus in condition (1), there is only one singular part to consider.

In $H^{2}$, the $B$-invariant subspace $\left(\vartheta H^{2}\right)^{\perp}$ is singly generated by the vector $f=$ $B \vartheta$. This next corollary is the analogue of this for $(\mathcal{K}, *)$.

Corollary 2.6. $I(\vartheta, E)^{\perp}=\bigvee\left\{B^{n} f: n \in \mathbb{N} \cup\{0\}\right\}$, where $f=B(K \mu)$ for $d \mu=$ $\vartheta d m+d \mu_{s}$ and $\mu_{s} \perp m$ with support equal to $E$. 
Here $\bigvee$ is the closed linear span in $(\mathcal{K}, *)$. This next corollary mimics what happens in the Bergman space setting. By a classical result of Smirnov [9, p. 39], $\mathcal{K} \subseteq H^{p}$ for all $0<p<1$, and so if our $B$-invariant subspace $\mathcal{M} \subseteq \mathcal{K}$ has the property that $\mathcal{M}_{\perp}$ is generated by $f$, i.e., $\mathcal{M}_{\perp}$ is the closed linear span (in $A$ ) of $z^{n} f(n \in \mathbb{N} \cup\{0\})$, then certainly $f \mathcal{M} \subseteq H^{p}$ for all $0<p<1$. If $f$ is sufficiently smooth, we get the stronger condition $f \mathcal{M} \subseteq H^{1}$ and even a bit more.

Theorem 2.7. Suppose $f \in A$ with $f^{\prime} \in H^{\infty}$. Let $E=f^{-1}(\{0\}) \cap \mathbb{T}$, and let $\vartheta_{f}$ be the inner factor of $f$. Then $K \mu \perp z^{n} f$ for all $n \in \mathbb{N} \cup\{0\}$ if and only if

(1) $f K \mu \in H^{1}$;

(2) $K \mu / \vartheta_{f}$ has an analytic continuation across $\mathbb{T} \backslash E$ to a function $F \in N^{+}\left(\mathbb{D}_{e}\right)$ with $F(\infty)=0$.

If $f \in A$ with $f^{\prime} \in H^{\infty}$, then the boundary zero set $E$ of $f$ satisfies the so-called Carleson condition: If $\left(I_{n}\right)$ is the sequence of arcs contiguous to $E$ on the circle, then $\sum_{n}\left|I_{n}\right| \log \left|I_{n}\right|>-\infty$. Thus, by Theorem 2.2, not every $B$-invariant subspace of $(\mathcal{K}, *)$ is singly generated by such an $f$.

Comments about the $B$-invariant subspaces of $(\mathcal{K},\|\cdot\|)$ appear at the end of this note.

\section{The PROOFS}

Proposition 3.1. Suppose $\vartheta F$ is a generator for $I(\vartheta, E)$ and $d \mu=\phi d m+d \mu_{s}$ as in (2.4). Then $K \mu \perp z^{n} \vartheta F$ for all $n \in \mathbb{N} \cup\{0\}$ if and only if $\phi \in \vartheta \overline{H_{0}^{1}}$ and $\mu_{s}$ is supported in $E$.

Proof. Suppose $K \mu \perp z^{n} \vartheta F$ for all $n \in \mathbb{N} \cup\{0\}$. Then, by (2.1),

$$
\int_{\mathbb{T}} \overline{\zeta^{n} \vartheta F}\left(\phi d m+d \mu_{s}\right)=0 \text { for all } n \in \mathbb{N} \cup\{0\} .
$$

From the F. and M. Riesz theorem, $\overline{\vartheta F} d \mu_{s}$ is the zero measure (and so $\mu_{s}$ is supported in $E$ ) and $\overline{\vartheta F} \phi=\bar{h} \in \overline{H_{0}^{1}}$. However, $\phi \bar{\vartheta}=\overline{h / F} \in \overline{N^{+}}$and has $L^{1}(m)$ boundary values, and so $\phi \bar{\vartheta} \in \overline{H_{0}^{1}}$ [9, p. 28]. The converse is obvious.

Proof of Theorem 2.5. We start by proving a somewhat weaker result: $K \mu \perp$ $I(E, \vartheta)$ if and only if $\mu_{s}$ is supported in $E$ and $K \mu / \vartheta$ has a pseudocontinuation across $\mathbb{T}$ belonging to $N^{+}\left(\mathbb{D}_{e}\right)$ and vanishing at infinity. Indeed, suppose $K \mu \perp I(\vartheta, E)$. By Proposition 3.1 we can assume $\mu$ takes the form

$$
d \mu=\phi d m+d \mu_{s}, \quad \phi \bar{\vartheta}=\bar{k} \in \overline{H_{0}^{1}}, \quad \operatorname{supp}\left(\mu_{s}\right) \subseteq E .
$$

Since $\bar{k} \in \overline{H_{0}^{1}}$, then $\bar{k}(1 / \bar{z})$ belongs to $H^{1}\left(\mathbb{D}_{e}\right)$ and vanishes at infinity. The inner function $\vartheta$ is defined on $\mathbb{D}_{e}$ by $\vartheta(z)=1 / \overline{\vartheta(1 / \bar{z})}$. The function

$$
\widehat{\mu}(z):=\int \frac{d \mu(\zeta)}{1-\bar{\zeta} z}, \quad z \in \mathbb{D}_{e}
$$

belongs to $H^{p}\left(\mathbb{D}_{e}\right)$ for all $0<p<1[\underline{9}$ p. 39] and so the function

$$
T_{\mu, \vartheta}(z):=\bar{k}(1 / \bar{z})+\frac{\widehat{\mu}(z)}{\vartheta(z)}, \quad z \in \mathbb{D}_{e}
$$


belongs to $N^{+}\left(\mathbb{D}_{e}\right)$ and vanishes at infinity. By Fatou's jump theoren 2 , the boundary function for $T_{\mu, \vartheta}$ is

$$
\frac{\phi}{\vartheta}(\zeta)+\frac{(K \mu)(\zeta)-\phi(\zeta)}{\vartheta(\zeta)}=\frac{K \mu}{\vartheta}(\zeta)
$$

for $m$-almost every $\zeta \in \mathbb{T}$. Thus $T_{\mu, \vartheta}$ is the pseudocontinuation of $K \mu / \vartheta$ of the desired type.

Conversely, suppose $d \mu=\phi d m+d \mu_{s}$, where $\phi \in L^{1}(m)$ and $\mu_{s}$ is supported in $E$, and $K \mu / \vartheta$ has a pseudocontinuation $G \in N^{+}\left(\mathbb{D}_{e}\right)$ with $G(\infty)=0$. Then, by Fatou's jump theorem,

$$
G(\zeta)=\lim _{r \rightarrow 1^{-}} \frac{K \mu}{\vartheta}(r \zeta)=\frac{\phi(\zeta)+\widehat{\mu}(\zeta)}{\vartheta(\zeta)} .
$$

Assuming for the moment that $\vartheta(0) \neq 0$, we conclude that $G-\widehat{\mu} / \vartheta \in N^{+}\left(\mathbb{D}_{e}\right)$ and vanishes at infinity. Then $\phi / \vartheta$ is the boundary function of a function from $N^{+}\left(\mathbb{D}_{e}\right)$ that vanishes at infinity. But since $\phi / \vartheta \in L^{1}(m)$, then $\phi / \vartheta \in \overline{H_{0}^{1}}$. If $\vartheta(0)=0$, then use the same argument with $\vartheta$ replaced by $\vartheta / z^{n}$ and $G$ replaced by $G / z^{n}$ for some positive integer $n$. Now apply Proposition 3.1.

Now we need to show that $K \mu$ has an analytic continuation to $\widehat{\mathbb{C}} \backslash\left(\sigma(\vartheta)^{*} \cup E\right)$. As mentioned earlier, this was originally shown by Korenblum in 13. Indeed, if $W \subseteq \widehat{\mathbb{C}} \backslash\left(\sigma(\vartheta)^{*} \cup E\right)$ is an open set containing an arc of the circle, then $T_{\mu, \vartheta}$ (as defined in (3.3) $)$ is analytic on $W \cap \mathbb{D}_{e}$ and by standard estimates,

$$
\left|T_{\mu, \vartheta}(\lambda)\right| \leqslant C\|\mu\| \frac{1}{|\lambda|-1}, \quad \lambda \in W \cap \mathbb{D}_{e} .
$$

Since $K \mu \perp I(\vartheta, E)$, we can apply Proposition 3.1 to conclude that $\mu$ takes the form

$$
d \mu=\phi d m+d \mu_{s},
$$

where $\phi=\vartheta \bar{h}\left(h \in H_{0}^{1}\right)$ and $\mu_{s}$ is supported in $E$.

Next, let $\left(h_{n}\right)$ be a sequence of polynomials in $H_{0}^{1}$ that approximates $h$ in norm and set

$$
d \mu_{n}:=\vartheta \overline{h_{n}} d m+d \mu_{s} .
$$

Notice that $\left\|\mu_{n}\right\|$ is uniformly bounded in $n$. By Proposition 3.1, $K \mu_{n} \perp I(\vartheta, E)$ and the corresponding pseudocontinuation of $K \mu_{n} / \vartheta$ is

$$
T_{\mu_{n}, \vartheta}(z)=\overline{h_{n}}(1 / \bar{z})+\frac{1}{\vartheta(z)} \int \frac{\vartheta(\zeta) \overline{h_{n}}(\zeta)}{1-\bar{\zeta} z} d m(\zeta)+\frac{1}{\vartheta(z)} \int \frac{d \mu_{s}(\zeta)}{1-\bar{\zeta} z}
$$

Since the functions $\vartheta \overline{h_{n}}$ are bounded on $\mathbb{T}$, then $K \mu_{n} / \vartheta$ and $T_{\mu_{n}, \vartheta}$ are $H^{1}$ functions on $W \cap \mathbb{D}$ and $W \cap \mathbb{D}_{e}$ (respectively) [9 p. 41]. (Note that $\vartheta$ has an analytic continuation across $W \cap \mathbb{T}$ as does $\widehat{\mu_{s}}$ since this $W \cap \mathbb{T}$ avoids the support of $\mu_{s}$.) Moreover, by what was said earlier, they have equal boundary values almost everywhere on $W \cap \mathbb{T}$. By a standard Morera's theorem argument [10, p. 95], these two functions are analytic continuations of each other across $W \cap \mathbb{T}$.

\footnotetext{
${ }^{2}$ Fatou's jump theorem: $\lim _{r \rightarrow 1^{-}}(\widehat{\mu}(r \zeta)-\widehat{\mu}(\zeta / r))=\lim _{r \rightarrow 1^{-}} \int P_{r \zeta} d \mu=d \mu / d m(\zeta) m$-almost everywhere [9, p. 4].
} 
Finally,

$$
\begin{gathered}
\left|\frac{K \mu_{n}}{\vartheta}(\lambda)\right| \leqslant C\left\|\mu_{n}\right\| \frac{1}{1-|\lambda|} \leqslant \frac{C}{1-|\lambda|}, \quad \lambda \in W \cap \mathbb{D}, \\
\left|T_{\mu_{n}, \vartheta}(\lambda)\right| \leqslant C\left\|\mu_{n}\right\| \frac{1}{|\lambda|-1} \leqslant \frac{C}{|\lambda|-1}, \quad \lambda \in W \cap \mathbb{D}_{e} .
\end{gathered}
$$

By a theorem of Beurling [7] (see also [15, p. 95]), these functions form a normal family on $W$ and so a subsequence (for which we will use the same index) converges to an analytic function on $W$. But since $K \mu_{n} / \vartheta$ converges pointwise to $K \mu / \vartheta$, then $K \mu / \vartheta$, and hence $K \mu$, will have an analytic continuation to $W$.

Proof of Corollary [2.6] If $\vartheta F$ is a generator of the ideal $I(\vartheta, E)$, then by our "Cauchy pairing" in (2.1), it is routine to show that

$$
\left\langle z^{m} \vartheta F, B^{n} f\right\rangle=\int_{\mathbb{T}} \overline{\vartheta F \zeta^{n+m+1}}\left(\vartheta d m+d \mu_{s}\right)=0 \quad \forall m, n \in \mathbb{N} \cup\{0\} .
$$

Thus

$$
\bigvee\left\{B^{n} f: n=0,1,2, \ldots\right\} \subseteq I(\vartheta, E)^{\perp} .
$$

If $g \in A$ satisfies $\left\langle g, B^{n} f\right\rangle=0$ for all $n$, one can use the F. and M. Riesz theorem to show that $g / \vartheta \in H^{1}$ and $g$ is zero on the support of $\mu_{s}$ (which equals $E$ ). Thus $g \in I(\vartheta, E)$. An application of the Hahn-Banach theorem completes the proof.

The proof of Theorem2.7 requires a few preliminaries. Notice that $K \mu \in L^{1}(d A)$, where $d A$ is the area measure on $\mathbb{D}$. This follows from Fubini's theorem and the fact that the integral

$$
\int_{\mathbb{D}} \frac{1}{\left|e^{i \theta}-z\right|} d A(z)
$$

is uniformly bounded in $\theta$.

For a Cauchy transform $K \mu$, consider the function

$$
\int_{\mathbb{D}} \frac{(K \mu)(z)}{z-\lambda} d A(z), \quad \lambda \in \mathbb{D} .
$$

Since $K \mu \in L^{1}(d A)$ and is analytic on $\mathbb{D}$, it is not difficult to show, using the fact that $(z-\lambda)^{-1} \in L^{1}(d A)$ for each fixed $\lambda \in \mathbb{D}$, that the above integral exists for every $\lambda \in \mathbb{D}$. Moreover, the dominated convergence theorem says that the above function is continuous on $\mathbb{D}$.

Proposition 3.4. For $\mu \in M$,

$$
\sup _{0<r<1} \int_{0}^{2 \pi} \int_{\mathbb{D}}\left|\frac{(K \mu)(z)}{z-r e^{i \theta}}\right| d A(z) d \theta<\infty .
$$

Proof. For fixed $0<r<1$,

$$
\begin{aligned}
& \int_{0}^{2 \pi} \int_{\mathbb{D}}\left|\frac{(K \mu)(z)}{z-r e^{i \theta}}\right| d A(z) d \theta \\
\leqslant & \int_{0}^{2 \pi} \int_{0}^{1} \int_{0}^{2 \pi} \int_{\mathbb{T}} \frac{1}{\left|s e^{i t}-r e^{i \theta}\right|} \frac{1}{\left|1-s e^{i t} e^{-i x}\right|} d|\mu|\left(e^{i x}\right) d t d s d \theta .
\end{aligned}
$$

Use the standard inequality

$$
\int_{0}^{2 \pi} \frac{d \theta}{\left|e^{i \theta}-a\right|} \leqslant C \log \left(\frac{1}{1-|a|}\right), \quad|a|<1
$$


to get

$$
\int_{0}^{2 \pi} \frac{d \theta}{\left|s e^{i t}-r e^{i \theta}\right|} \leqslant C \begin{cases}\frac{1}{r} \log \left(\frac{1}{1-s / r}\right) & \text { for } s<r \\ \frac{1}{r} \log \left(\frac{1}{1-r / s}\right) & \text { for } s>r\end{cases}
$$

and

$$
\int_{0}^{2 \pi} \frac{d t}{\left|1-s e^{i t} e^{-i x}\right|} \leqslant C \log \left(\frac{1}{1-s}\right) .
$$

Combine the above two estimates along with Fubini's theorem to show the desired integral is bounded above by

$$
\frac{C}{r}\left[\int_{0}^{r} \log \left(\frac{1}{1-s}\right) \log \left(\frac{1}{1-s / r}\right) d s+\int_{r}^{1} \log \left(\frac{1}{1-s}\right) \log \left(\frac{1}{1-r / s}\right) d s\right] .
$$

Standard estimates now show that this quantity is bounded uniformly for $r$ close to 1 .

Proof of Theorem 2.7. Suppose $f \in A$ with $f^{\prime} \in H^{\infty}$ and $K \mu \perp z^{n} f$ for all $n \in$ $\mathbb{N} \cup\{0\}$. Theorem 2.5 yields condition (2). Using a power series argument, one can show that

$$
\langle f, K \mu\rangle=\lim _{r \rightarrow 1^{-}} \sum_{n=0}^{\infty} \overline{\widehat{f}(n)} \widehat{\mu}(n) r^{n}=\lim _{r \rightarrow 1^{-}} \int_{\mathbb{D}}(K \mu)(r z) \overline{(z f)^{\prime}(r z)} d m_{2}(z),
$$

where $d m_{2}=d A / \pi$. Since $(z f)^{\prime}$ is a bounded function, we can use the fact that $K \mu \in L^{1}(d A)$, to rewrite ${ }^{3}$ this as

$$
\int_{\mathbb{D}}(K \mu)(z) \overline{(z f)^{\prime}(z)} d m_{2}(z) .
$$

For fixed $\lambda \in \mathbb{D}$, the function

$$
\frac{K \mu-(K \mu)(\lambda)}{z-\lambda}
$$

can be written as $K \mu_{\lambda}$, where $d \mu_{\lambda}=\bar{\zeta}(1-\bar{\zeta} \lambda)^{-1} d \mu$. By Proposition [3.1, $K \mu_{\lambda}$ also annihilates the ideal generated by $f=\vartheta F$. Thus, by what was said above,

$$
\int_{\mathbb{D}} \frac{(K \mu)(z)-(K \mu)(\lambda)}{z-\lambda} \overline{(z f)^{\prime}(z)} d m_{2}(z)=0, \quad \lambda \in \mathbb{D} .
$$

Another power series computation yields

$$
\int_{\mathbb{D}} \frac{\overline{(z f)^{\prime}(z)}}{z-\lambda} d m_{2}(z)=-\overline{\lambda f(\lambda)}
$$

and so from (3.5),

$$
-\overline{\lambda f(\lambda)}(K \mu)(\lambda)=\int_{\mathbb{D}} \frac{(K \mu)(z)}{z-\lambda} \overline{(z f)^{\prime}(z)} d m_{2}(z) .
$$

Now use Proposition 3.4 along with the assumption that $(z f)^{\prime}$ is bounded to show that the integrals

$$
\int_{0}^{2 \pi}\left|f\left(r e^{i \theta}\right)(K \mu)\left(r e^{i \theta}\right)\right| d \theta
$$

are uniformly bounded in $0<r<1$, that is to say, $f K \mu \in H^{1}$.

\footnotetext{
${ }^{3}$ See, for example, the argument used to prove Lemma 2.5 in [3].
} 
Conversely, suppose conditions (1) and (2) are satisfied. Since $\overline{\vartheta_{f}} K \mu$ and $\overline{F_{f}}$ (where $F_{f}$ is the outer factor of $f$ ) are the boundary values of functions from $N^{+}\left(\mathbb{D}_{e}\right)$, then $\bar{f} K \mu$ is also the boundary function of a $N^{+}\left(\mathbb{D}_{e}\right)$ function that vanishes at infinity. But since $\bar{f} K \mu \in L^{1}(m)$, then $\bar{f} K \mu \in \overline{H_{0}^{1}}$. Thus

$$
\int_{\mathbb{T}}(K \mu)(\zeta) \overline{\zeta^{n} f(\zeta)} d m(\zeta)=0 \text { for all } n \in \mathbb{N} \cup\{0\} .
$$

Finally, using the notation $g_{r}(z):=g(r z)$,

$$
(K \mu)_{r} \overline{f_{r}}-K \mu \bar{f}=\left[(K \mu)_{r} f_{r}-K \mu f\right] \overline{\frac{f_{r}}{f_{r}}}+K \mu f\left[\frac{\overline{f_{r}}}{f_{r}}-\bar{f}\right],
$$

which goes to zero in the $L^{1}(m)$ norm as $r \rightarrow 1^{-}$. Thus for any $n \in \mathbb{N} \cup\{0\}$,

$$
\begin{aligned}
\left\langle z^{n} f, K \mu\right\rangle & =\lim _{r \rightarrow 1^{-}} \int_{\mathbb{T}}(K \mu)(r \zeta) \overline{(r \zeta)^{n} f(r \zeta)} d m(\zeta) \\
& =\int_{\mathbb{T}}(K \mu)(\zeta) \overline{\zeta^{n} f(\zeta)} d m(\zeta) \\
& =0
\end{aligned}
$$

\section{THE NORM TOPOLOGY}

Recall that $(\mathcal{K},\|\cdot\|)$ is a nonseparable space, and so a characterization of the $B$-invariant subspaces is out of reach. In this final section, we will make a few remarks about the subspace $[K \mu]$, which we define to be the smallest $B$-invariant subspace of $(\mathcal{K},\|\cdot\|)$ containing $K \mu$.

By the Lebesgue decomposition theorem, the space of measures can be decomposed as $M=M_{a} \oplus M_{s}$, where $M_{a}=\left\{\phi d m: \phi \in L^{1}(m)\right\}$ (the absolutely continuous measures with respect to Lebesgue measure $m$ ) and $M_{s}=\{\mu \in M: \mu \perp m\}$ (the singular ones). Moreover, if $\mu=\mu_{a}+\mu_{s}\left(\mu_{a} \in M_{a}, \mu_{s} \in M_{s}\right)$, then

$$
\|\mu\|=\left\|\mu_{a}\right\|+\left\|\mu_{s}\right\| \text {. }
$$

As a consequence of this, the space $(\mathcal{K},\|\cdot\|)$ can be decomposed as $\mathcal{K}=\mathcal{K}_{a} \oplus \mathcal{K}_{s}$, where $\mathcal{K}_{a}=\left\{K(\phi d m): \phi \in L^{1}(m)\right\}$ and $\mathcal{K}_{s}=\{K \mu: \mu \perp m\}$. One can show that $\mathcal{K} \simeq M / \overline{H_{0}^{1}}$ (where $\overline{H_{0}^{1}}$ is equated with a subspace of $M_{a}$ in the obvious way) and $\mathcal{K}_{a} \simeq L^{1} \overline{H_{0}^{1}}$. This makes the space $\left(\mathcal{K}_{a},\|\cdot\|\right)$ separable. See [4], 5], and [6] for details.

Although the $B$-invariant subspaces of $(\mathcal{K},\|\cdot\|)$ are very much unknown (due to the nonseparability of $\left.\mathcal{K}_{s}\right)$, the $B$-invariant subspaces of $\left(\mathcal{K}_{a},\|\cdot\|\right)$ are known [1] (see also [6, p. 99]).

Theorem 4.2 (Aleksandrov). If $\mathcal{M}$ is a B-invariant subspace of $\left(\mathcal{K}_{a},\|\cdot\|\right)$, then there is an inner function $\vartheta$ such that $f \in \mathcal{M}$ if and only if $f / \vartheta$ has a pseudocontinuation across $\mathbb{T}$ to a function belonging to $N^{+}\left(\mathbb{D}_{e}\right)$ and vanishing at infinity.

We now examine $[K \mu]$ (the smallest $B$-invariant subspace of $(\mathcal{K},\|\cdot\|)$ containing $K \mu$ ), where $\mu \in \mathcal{M}$ and whose support is not all of $\mathbb{T}$. First notice the following.

Proposition 4.3. If $\mu \in M \backslash\{0\}$ with $\mu \ll m$ and $\operatorname{supp}(\mu) \neq \mathbb{T}$, then $[K \mu]=\mathcal{K}_{a}$. 
Proof. Indeed, if the support of $\mu$ omits the $\operatorname{arc} J \subseteq \mathbb{T}$, then $K \mu$ has an analytic continuation across $J$ given by

$$
\widehat{\mu}(z)=\int_{\mathbb{T}} \frac{d \mu(\zeta)}{1-\bar{\zeta} z}, \quad z \in \mathbb{D}_{e} .
$$

Moreover, if $[K \mu] \neq \mathcal{K}_{a}$, then by Aleksandrov's theorem, $K \mu / \vartheta$ will have a pseudocontinuation for some inner function $\vartheta$. But since any inner function $\vartheta$ has a pseudocontinuation given by

$$
\widetilde{\vartheta}(z)=\frac{1}{\overline{\vartheta(1 / \bar{z})}}, \quad z \in \mathbb{D}_{e},
$$

then $K \mu$ will have a pseudocontinuation $F$. That is to say, $F$ is meromorphic on $\mathbb{D}_{e}$ and has nontangential boundary values equal to those of $K \mu \mathrm{m}$-almost everywhere on $\mathbb{T}$. So there are two meromorphic functions on $\mathbb{D}_{e}$, namely $F$ and $\widehat{\mu}$, that have nontangential boundary values equal to $K \mu \mathrm{m}$-almost everywhere on the arc $J$. By Privalov's uniqueness theorem [12] pp. $62-63] 4, F=\widehat{\mu}$. Thus $\widehat{\mu}$ is a pseudocontinuation of $K \mu$ across $\mathbb{T}$. So

$$
\lim _{r \rightarrow 1^{-}}[(K \mu)(r \zeta)-\widehat{\mu}(\zeta / r)]=0
$$

for $m$-almost every $\zeta$. By Fatou's jump theorem and the absolute continuity of $\mu$, $\mu$ must be the zero measure, a contradiction.

If $p$ is an analytic polynomial, then $p(B) K \mu=K(p(\bar{\zeta}) d \mu)$. Assuming $\operatorname{supp}(\mu) \neq$ $\mathbb{T}$, we can apply Mergelyan's theorem [17, p. 423] along with the density of the continuous functions in $L^{1}(\mu)$ as well as the inequality $\|K \mu\| \leqslant\|\mu\|$, to conclude that

$$
[K \mu]=\operatorname{clos}_{\mathcal{K}}\left\{K(f d \mu): f \in L^{1}(\mu)\right\} .
$$

Recall from the definition of the norm and (4.1) that for $\mu \in M_{s},\|K \mu\|=\|\mu\|$. It follows now from (4.4) that for $\mu \perp m$ and $\operatorname{supp}(\mu) \neq \mathbb{T}$,

$$
[K \mu]=\left\{K(f d \mu): f \in L^{1}(\mu)\right\} .
$$

If $\mu_{1} \ll \mu_{2}$ with $\operatorname{supp}\left(\mu_{2}\right) \neq \mathbb{T}$, then $d \mu_{1}=g d \mu_{2}$, where $g \in L^{1}\left(\mu_{2}\right)$. Thus if $f \in L^{1}\left(\mu_{1}\right)$, then $K\left(f d \mu_{1}\right)=K\left(f g d \mu_{2}\right)$ and so by (4.4), we have shown the following.

Proposition 4.6. If $\mu_{1} \ll \mu_{2}$ and $\operatorname{supp}\left(\mu_{2}\right) \neq \mathbb{T}$, then $\left[K \mu_{1}\right] \subseteq\left[K \mu_{2}\right]$.

If $\mu \in M$ and is positive with $\operatorname{supp}(\mu) \neq \mathbb{T}$, and $\mu=\mu_{a}+\mu_{s}\left(\mu_{a} \in M_{a}\right.$ and $\mu_{s} \in M_{s}$ ), we note that $\mu_{a} \ll \mu$ and $\mu_{s} \ll \mu$. We can now apply Proposition 4.6 along with (4.5) and Proposition 4.3 to obtain the following result.

Theorem 4.7. If $\mu \in M \backslash\{0\}$ is positive with $\operatorname{supp}(\mu) \neq \mathbb{T}$ and $\mu=\mu_{a}+\mu_{s}$, then

$$
[K \mu]= \begin{cases}\mathcal{K}_{a} \oplus\left\{K\left(f d \mu_{s}\right): f \in L^{1}\left(\mu_{s}\right)\right\} & \text { if } \mu_{a} \neq \equiv 0 \\ \left\{K\left(f d \mu_{s}\right): f \in L^{1}\left(\mu_{s}\right)\right\} & \text { if } \mu_{a} \equiv 0 .\end{cases}
$$

\footnotetext{
${ }^{4}$ Privalov's uniqueness theorem: If $f$ is meromorphic on $\mathbb{D}$ and has nontangential limits that exist and are equal to zero on a set of positive measure in $\mathbb{T}$, then $f$ is the zero function.
} 


\section{REFERENCES}

1. A. B. Aleksandrov, Invariant subspaces of shift operators. An axiomatic approach, Zap. Nauchn. Sem. Leningrad. Otdel. Mat. Inst. Steklov. (LOMI) 113 (1981), 7-26, 264, Investigations on linear operators and the theory of functions, XI. MR 83g:47031

2. A. Aleman and W. T. Ross, The backward shift on weighted Bergman spaces, Michigan Math. J. 43 (1996), no. 2, 291-319. MR 97i:47053

3. S. Axler, Bergman spaces and their operators, Surveys of some recent results in operator theory, Vol. I, Longman Sci. Tech., Harlow, 1988, pp. 1-50. MR 90b:47048

4. P. Bourdon and J. A. Cima, On integrals of Cauchy-Stieltjes type, Houston J. Math. 14 (1988), no. 4, 465-474. MR 90h:30095

5. J. A. Cima, A. Matheson, and W. T. Ross, The Cauchy transform, preprint.

6. J. A. Cima and W. T. Ross, The backward shift on the Hardy space, American Mathematical Society, Providence, RI, 2000. MR 2002f:47068

7. Y. Domar, On the existence of a largest subharmonic minorant of a given function, Ark. Mat. 3 (1957), 429-440. MR 19:408c

8. R. G. Douglas, H. S. Shapiro, and A. L. Shields, Cyclic vectors and invariant subspaces for the backward shift operator, Ann. Inst. Fourier (Grenoble) 20 (1970), fasc. 1, 37-76. MR 42:5088

9. P. L. Duren, Theory of $H^{p}$ spaces, Academic Press, New York, 1970. MR 42:3552

10. J. B. Garnett, Bounded analytic functions, Academic Press Inc., New York, 1981. MR 83g:30037

11. K. Hoffman, Banach spaces of analytic functions, Dover Publications Inc., New York, 1988, Reprint of the 1962 original. MR 92d:46066

12. P. Koosis, Introduction to $H_{p}$ spaces, second ed., Cambridge University Press, Cambridge, 1998. MR 2000b:30052

13. B. I. Korenblum, Closed ideals of the ring $A^{n}$, Funkcional. Anal. i Priložen. 6 (1972), no. 3, 38-52. MR 48:2776

14. S. Richter and C. Sundberg, Invariant subspaces of the Dirichlet shift and pseudocontinuations, Trans. Amer. Math. Soc. 341 (1994), no. 2, 863-879. MR 94d:47026

15. W. T. Ross and H. S. Shapiro, Generalized analytic continuation, University Lecture Series, vol. 25, American Mathematical Society, Providence, RI, 2002. MR 2003h:30003

16. W. Rudin, The closed ideals in an algebra of analytic functions, Canad. J. Math. 9 (1957), 426-434. MR 19:641c

17. $ـ$ Real and complex analysis, second ed., McGraw-Hill Series in Higher Mathematics, McGraw-Hill Book Co., New York, 1974. MR 49:8783

Department of Mathematics, University of North Carolina, Chapel Hill, North CarOLINA 27599

E-mail address: cima@math.unc.edu

Department of Mathematics, Lamar University, Beaumont, Texas 77710

E-mail address: matheson@math.lamar.edu

Department of Mathematics and Computer Science, University of Richmond, RichMOND, VIRGINIA 23173

E-mail address: wross@richmond.edu 